\title{
Integrating Semantic Technologies with Interactive Digital TV
}

\author{
Antonis Papadimitriou, Christos Anagnostopoulos, Vassileios Tsetsos, Sarantis \\ Paskalis and Stathes Hadjiefthymiades \\ Pervasive Computing Research Group, Department of Informatics and \\ Telecommunications, University of Athens, Panepistimioupolis 15784, Athens, \\ Greece, $\{$ anthony, bleu, b.tsetsos, paskalis, shadj\}@di.uoa.gr
}

\begin{abstract}
Interactive digital TV is becoming a reality throughout the globe. The most essential aspect of TV broadcasting is enhancing the interacting experience for the viewer. To this end, we explore the potential of introducing semantics in the distribution, processing and usage of the media content. We propose a smart iTV receiver framework capable of collecting, extending and processing semantic metadata related to the broadcast multimedia content. System architecture is presented along with examples of services to illustrate the combination of semantic metadata content, user preferences and external data sources.
\end{abstract}

\section{Introduction}

There is no doubt that Television (TV) is one of the most prevalent media technologies. Analog TV broadcasts have been on air for more than five decades, and their impact has qualified TV as a massively used technology throughout the globe. Yet, recent technological developments have created new opportunities for the evolvement of the TV Broadcasting market. Technological advances include the appearance of Digital TV (DTV) and the manufacturing of Set-Top-Boxes (STBs) capable of executing interactive applications in the TV receiver. To this end, over the past years, there has been a coordinated effort from multiple organizations towards the establishment of standard digital transmission technologies and application execution environments for interactive TV (iTV). This has led to the development of several standards such as Digital Video Broadcasting (DVB) transmission specifications [6] (for satellite, cable and terrestrial TV) and the Multimedia Home Platform (MHP) [3] as middleware for interoperable interactive applications.

Most interestingly, there is already an extensive network of digital TV (DTV) infrastructure based on these specifications, numbering a multitude of homes subscribed to iTV services from different broadcasters. Hence, an open market is evolving with great prospects of benefit both in terms of provider profit and user satis- 
faction. The next step to the future of digital TV is the enhancement of interactive applications provided to the iTV subscribers. This is critical for the consolidation of iTV and its further penetration into the home entertainment market. Towards the goal of providing rich and personalized interactive services we propose a semanticsaware iTV receiver platform and the respective business model for its exploitation. The proposed platform is named POLYSEMA and features a prototype iTV receiver system capable of collecting, extending and processing semantic metadata related to the broadcast multimedia content.

Metadata information is used at the receiver in order to offer pioneer services to users. One such service is the retrieval of web information related to the current TV program. For instance, a user might have included in their profile that they are specifically interested in some movie star. Then, every time that particular actor appears on a TV program (movie, show etc.) the system can retrieve from the web information, trivia and news about that actor. Another example is allowing for a finer granularity of parental control over the media content shown on TV. Specifically, parents may designate some rules about the content their children are forbidden to watch and each time the metadata describing a video scene triggers the respective rules, the display of audiovisual content on the screen will be suppressed. Other interactive services may include smart personal video recording, semantic channel recommendation and many more.

The technical specification of the POLYSEMA platform is based on several standards widely adopted by research and industry communities. This makes the proposed system a compelling solution for STB manufacturers and TV Broadcasters worldwide. Particularly, the standards fostered by POLYSEMA include the DVB$\mathrm{T}$ specifications [5], the MHP standard as an application execution environment [3], the OSGi platform for a service-oriented integration platform [1], the MPEG-7 standard as a metadata description standard [17] and the Semantic Web technologies [10] as the base for semantics representation and logic-based inference.

The contribution of our work is manifold. To our knowledge, previous work on semantic applications for interactive TV focused on particular services such as channel recommendation [12]. On the contrary, we consider the development of a broad range of semantically enriched applications, all built upon an extensible set of basic services offered by the core POLYSEMA platform. Moreover, we introduce the concept of Scene-by-Scene interactive TV, which stands for the development of applications which adapt their behavior according to the content shown during each separate video scene. Another novelty of our system is that users can modify a wide set of preferences and define rules about when applications should be triggered, thus leading to an increased level of personalization. An additional critical feature of the design of the POLYSEMA receiver is that it supports the development of applications which can retrieve and display program-relevant information from the World Wide Web. This is an attempt towards the convergence of iTV with the WWW, a combination which can leverage the quality and broaden the scope of services offered in the context of iTV.

Lastly, we substantiate that our prototype system can be easily migrated to the real world of broadcasting by describing a flexible business model which allows for 
a multitude of value-added services from multiple providers. In this business model, media providers have to supply multimedia content, whereas service and metadata providers provide interactive services and metadata describing the content in some certain format (MPEG-7 [17]). Broadcasters multiplex interactive applications into their transport streams so that TV viewers receive both the multimedia content and the application logic required to take advantage of metadata descriptions. Receivers download metadata from the transport stream and/or the web sites of service aggregators, and process the information to match displayed content with user-defined rules and preferences.

The rest of the paper is organized as follows: Section 2 presents the work related to the POLYSEMA approach. In Section 3, we elaborate on the architecture of the proposed system, whereas Section 4 illustrates examples of interactive services and how they can be supported by the proposed iTV Platform. Section 5 is used to describe the business model which allows multiple stakeholders participate in the media content distribution value chain and thus hold a share in the revenue of the iTV market. Finally, section 6 concludes the chapter.

\section{Related Work}

Since the appearance of digital TV both academic and industrial stakeholders have focused on the convergence of the Web with the world of television broadcasting. The DVB consortium [6] produced the specifications of the Multimedia Home Platform [3], in order to establish the features that a state-of-the-art interactive television platform should support. The MHP specification includes a profile which supports web browsing and email client functionality. Web browsing is realized via the introduction of the DVB-HTML [14] markup language. Unfortunately, DVB-HTML has not been adopted yet by STB manufacturers because it is a quite complex specification, thus leading to higher device costs and the rise of interoperability considerations. An alternative to DVB-HTML was recently presented in [13]. That work developed a method for transcoding web pages to MHP-compliant visual components. This meant that even STBs with lower capabilities can browse the web.

Nevertheless, convergence of WWW with DTV does not only refer to browsing the web from the television screen and remote control. It also signifies that a broad scope of diverse applications can be migrated from the web to the TV broadcasting world. Especially when it comes to personalized and smart interactive services, semantic web can be the key technology to enable the development of pioneer multimedia platforms. Two major research efforts towards this direction are the AVATAR [12] and the MediaNet [18] projects. AVATAR is a project which utilizes most of the techniques and technologies that the POLYSEMA project uses, such as video annotation, ontology-based modeling, multimedia metadata, and user profiling/personalization through semantic reasoning. The project's main objective though is to create a personalized digital TV program recommender. The system accomplishes the task by taking into account the user's profile and TV-Anytime 
[8] meta-information of each TV program. The POLYSEMA project, on the other hand, provides a platform which supports a variety of personalized interactive services. The MediaNET project is divided in five sub-projects, each of which covers a significant area of the multimedia content: creation, service providers, network operators, etc. As far as interactive services are concerned, it delivers the AmigoTV service and a PVR. Moreover, MediaNET offers iTV/Web convergent services such as e-voting and e-shopping via the TV device. However, the project does not investigate the benefits from using metadata during the various phases of the multimedia content lifecycle.

Another aspect in designing iTV platforms based on MHP is how applications running on the MHP runtime environment can communicate with the rest of the system. The work introduced in [21], consists of a low-level implementation of a platform, which is both OSGi and MHP compliant. It uses the notion of XbundLets, i.e. classes that implement both the interface of an OSGi bundle and the MHP application lifecycle. XBundLets feature several advantages, such as straightforward bi-directional communication between OSGi Bundles and MHP Xlets, as well as improved performance. On the other hand, to support such a dual execution environment, the reference implementation of the MHP platform needs to be modified. This is a major drawback in adopting this architectural approach, as protocol compliance is a crucial issue in Interactive Television.

\section{POLYSEMA Architecture}

The goal of providing semantic-awareness in iTV services demands the cooperation of several diverse information and telecommunication systems. Specifically, digital broadcasting systems have to be combined with knowledge-based systems such as reasoners and rule engines. In a task like this, the architecture of POLYSEMA platform had to be carefully designed in order to be modular enough, so that the complexity of the implementation could be kept manageable.

Another important consideration in the development of the platform was that it had to be compatible with current broadcasting technologies and standards adopted world-wide. This feature facilitates future attempts for seamless integration of semantic-aware services into the real world of Interactive TV. Nevertheless, attaining provision of innovative services without compromising compatibility with existing products is not a trivial matter.

This section is devoted to describing the modular architecture of POLYSEMA platform by presenting the novel design of the receiver, as well as the enhancements at the broadcasting server. Moreover, we explain how POLYSEMA software components cooperate with existing broadcasting and receiver solutions. 


\subsection{Extending the Broadcasting Server}

MPEG-7 files can describe multimedia content on a scene-by-scene basis, by providing distinct descriptions for different sections of the video separated by clearly defined time markers. This model allows for a wide range of interactive applications, which adjust their behavior as the content presentation advances. A remarkable problem inhibiting such applications is that, in broadcast environments, the receiver cannot keep track of the absolute media time of individual TV programs in the incomming Transport Stream. That happens because iTV subscribers may tune to a specific program at any time during the broadcast. In such cases, applications are unaware of the absolute media time of a program event. The only way to synchronize video with an application is to transmit stream events within the stream, as described in the DSM-CC specification [2].

To overcome this problem, POLYSEMA utilizes a software module to convert MPEG-7 timestamps to stream events sent by the broadcasting server. The module parses the MPEG-7 document, which describes the content to be broadcast, and performs a mapping of MPEG-7 time elements to stream events, at the granularity of video segments. A time event signals the transition to a new scene of the video, which is associated to an MPEG-7 segment. A stream event consists of an identifier, name and data. Data refers to any string, so it is possible that the data field may contain the MPEG-7 segment identifier. Any time the receiver tunes to a channel, the application can determine which part of the video is being displayed by listening for stream events and consulting the MPEG-7 document.

Summing up, the Transport Stream (TS) produced at the server side and sent to the receiver contains:

- AV content, MHP applications, and the broadcast file system mounted on the transport stream,

- stream event objects that convey synchronization information,

- files in the DSM-CC object carousel, which may include MPEG-7 files describing the multimedia content or links to those MPEG-7 files, so that the receiver can locate and obtain the corresponding metadata files over the Internet.

As one can see in the above list, the MPEG-7 document (or a URL link to it) is sent over the Broadcast File System. Recent work on carrying metadata in MPEG-2 Streams (see [11] for a comprehensive presentation of the respective ISO amendment) defines different methods for this purpose. Metadata can be sent either by using private sections of MPEG-2, Packetized Elementary Stream (PES) packets or the Broadcast File System. The latter approach was preferred in POLYSEMA, as it allows for prefetching of the complete metadata, so that it can be timely preprocessed by the semantic component, before the respective video scenes arrive. Moreover, the POLYSEMA receiver can retrieve the metadata from the Internet, by reading just a URL link from the broadcast filesystem. We believe that this model of metadata transmission is more appropriate, because it saves bandwidth for AV information in the TS, while the metadata can be fetched concurrently from an Internet connection available at the receiver. 


\subsection{The POLYSEMA Smart Receiver Architecture}

Each interactive service consists of both a presentation part and a logic part which run at the Multimedia and the Semantic Component of POLYSEMA respectively (Fig. 1). Presentation refers to actions that the media player (i.e. DVB-MHP receiver) should perform when it comes to displaying the results of the logic part. Such actions are implemented in a platform-independent way by using the Javabased MHP application environment. The Semantic Component determines the actions which comprise each service, i.e. the logic behind each interactive service. The Content Retrieval and Composition Component (CRC) is in charge of retrieving and integrating any external resources to be used by invoked services. The Service Management Component is responsible for allowing TV viewers to declare their preferences and define customized services, so that the Semantic Component will know when and what actions to trigger. All components are built in an OSGi-based service-oriented fashion, in order to provide maximum flexibility in composing interactive TV services. The rest of the section describes the details of each component.

\subsubsection{The Multimedia Component}

The Multimedia Component of the POLYSEMA platform refers to the set of applications running in the MHP Execution Environment of the DVB receiver. There is no need for specialized hardware, as any DVB-MHP receiver can download and run the MHP applications which comprise the Multimedia Component. The only requirement is that the MHP profile running at the STB provides for Return Chan-

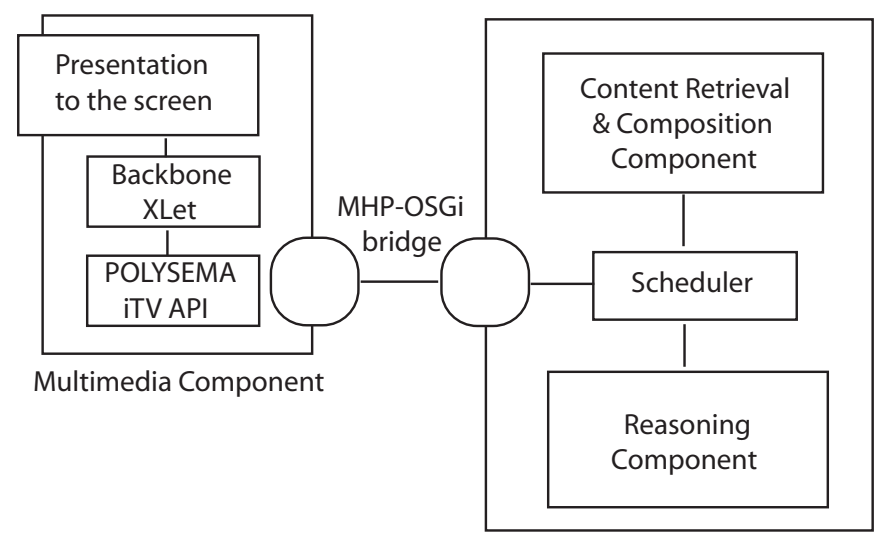

Semantic Component

Fig. 1 Overall system architecture of the POLYSEMA platform. 
nel (RC) communication. This is necessary for the interaction of the Multimedia Component with the Semantic Component.

The operation of the Multimedia Component is based on a basic MHP application (Xlet) running at the receiver. Xlets are classes that run under the JavaTV application model, which enforces a certain application lifecycle for every Xlet and an application manager that asserts the correct execution of many Xlets in one STB. The first responsibility of the basic Xlet of POLYSEMA is reading the broadcast file system and retrieving the MPEG-7 file describing the multimedia content (or the URL link pointing at locations where the metadata file is publicly available). The link or file is then fed to the Semantic Component which is accountable for storing and processing the semantic description. Moreover, the basic Xlet subscribes and listens to incoming stream events. Upon the reception of a stream event, it inquires the Semantic Component for actions that should be carried out for the specific video scene. Each action is implemented as a separate Java class, whose instances are created by the basic Xlet.

The complete set of available actions comprises the POLYSEMA iTV Application Programming Interface (API). This set includes methods/actions such as displaying messages and information, changing channel or sound volume, recording the video or hiding the displayed content. The API is described by the Service Description XML file which is downloaded by the Semantic Component from the Service Aggregator, as will be explained in a subsequent section. This file is used by the Service Management Component to let users combine basic actions and define customized services. The POLYSEMA iTV API is also used by the Semantic Component, in order to instruct the Multimedia Component about which actions to perform. Receivers that do not have semantic processing capabilities will still be able to display the multimedia content, but will not enjoy semantics-aware interactive services. The Multimedia Component also interacts with a local HTTP Server, running as an OSGi bundle, which is used as a proxy to store prefetched information retrieved from the WWW.

\subsubsection{The Semantic Component}

The Semantic Component includes all processes relevant to service metadata (i.e., inference processes that drive the personalized service provisioning) and the coordination of value added services offered by the platform. A more detailed view of its internal architecture is presented in Fig.2. A basic assumption for our system is that every AV content item is described by an MPEG-7 document. In order to reason over this document we transform it to a corresponding ontology, which was based on that proposed in [19]. In fact, we have developed a stripped down version of the ontology in order to eliminate any elements not used by our system. Moreover, the user defines, through the Service Management Component, their service preferences by combining templates of possible actions and declaring rules about when such services should launch and how they should be presented. The user input is based on the TV User Ontology, which describes the user profile and preferences. 
The Reasoning Component of the system uses the MPEG-7 document of the TV program and the user ontology (along with other domain ontologies such as the TV-Anytime classification schemes of MPEG-7) to infer which services should be activated during the broadcast. The Reasoning Component wraps the functionality of both a reasoning and a rule engine. Its reasoning capability is required mainly for classifying the multimedia content and the user preferences to predefined categories, while the rule support is used for deciding which services should be executed given the TV program metadata and the user profile. Bossam [15] is used for the implementation of the reasoning component. Once the appropriate services have been selected for execution by the Reasoning component (i.e., an action plan is formed), it is the responsibility of the Scheduler component to coordinate the execution of the respective application logic. Such logic is registered in the Service Registry module through procedures specified by OSGi.

The Scheduler instructs the Content Retrieval \& Composition (CRC) and the Multimedia components what actions they should perform. The Content Retrieval \& Composition component is a framework for registering and managing interfaces with external information sources. For each new source that is registered (e.g., Web site, multimedia database, RSS feed), the available content is described along with its type (e.g., text, video) and the invocation details (e.g., source URL, parameters).

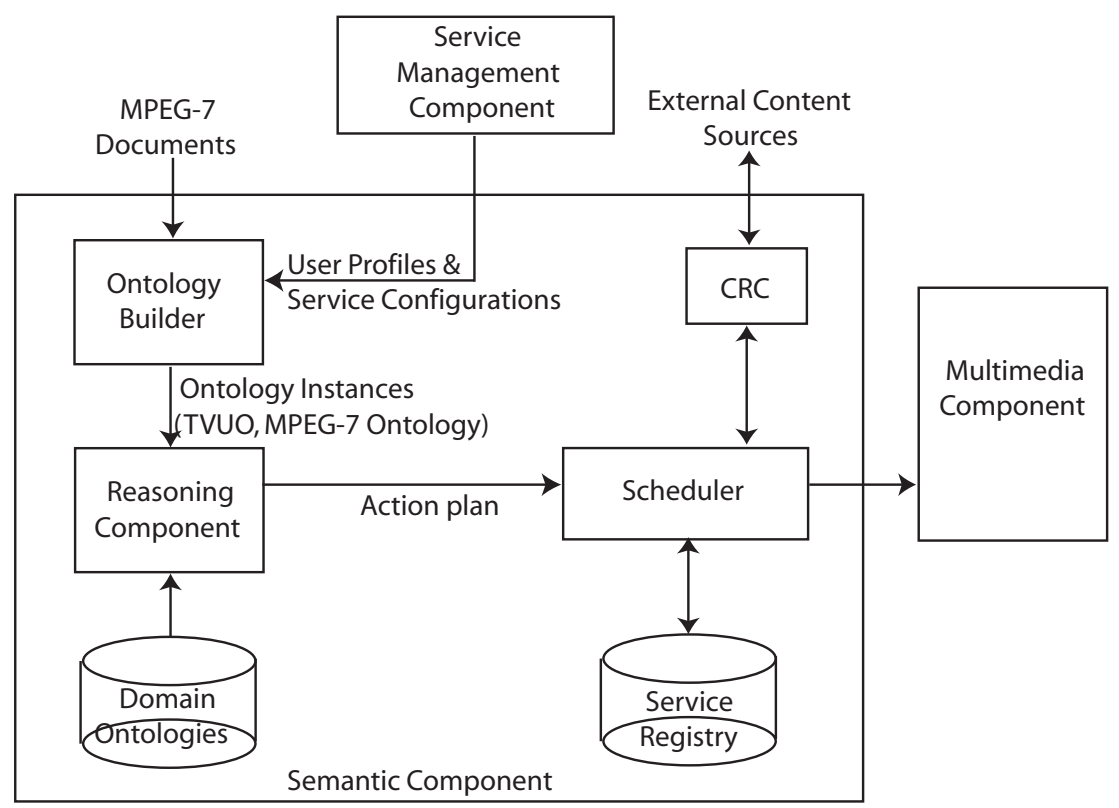

Fig. 2 Overall system architecture of the POLYSEMA platform. 


\subsubsection{Content Retrieval \& Composition Component}

The purpose of the Content Retrieval \& Composition Component (CRC) is to support the interactive services that display relevant information from the WWW. Once the Semantic Component has preprocessed the semantic description and has decided that certain web information should be fetched and displayed to the viewer, it instructs the CRC component to start prefetching the relevant data.

The CRC component consists of several bundles that implement the automated access to external web sites. For instance, in the prototype system we developed bundles which access content from several well-known sites such as IMDB [7] and Wikipedia [9]. The automated access classes follow a common abstract design, which involves management of created class instances, caching retrieved data till the end of the TV program and creating output lists containing all the requested web information.

The automated access bundles of various sites differ in the actual information available from these sites. This is reflected to the methods supported by different bundles. Each installed bundle updates a registry, called CRC Registry, which is used by the Scheduler of the Semantic Component to map user requested information to bundle method calls. Every call issued to the CRC component by the scheduler results in a piece of information retrieved and being cached in the internal structures of CRC bundles. In order to make this information available for display at the Multimedia Component, the CRC component returns the information to the Scheduler which then stores it in text or image files at the local HTTP Server running within the Semantic Component. The Multimedia Component then accesses the HTTP Server to retrieve the information files and display the content to the user.

The data fetched from the WWW can be either of textual or visual format. Text data may include movies information, encyclopedic knowledge, sport statistics etc. Visual information refers to images and photos related to a subject requested by the user.

\subsubsection{Service Management Component}

This component implements a management console for the POLYSEMA services. Specifically, it provides the users a graphical user interface (GUI) through which they can configure all the installed services according to their preferences. Since each residential gateway may have different services installed, this GUI cannot be predefined and delivered as a pre-built component. Hence, a dynamic GUI generation process is involved that automatically generates the GUI based on the service descriptions that accompany the service code. Moreover, the users can fill in their profiles though this component. The synthesis and rendering of the component's GUI is handled by Java Server Pages. The service configurations correspond to rules that should be applied to the displayed content and that define actions that should be taken by the other components (e.g., CRC) or by the MHP applications. Once the service preferences and user profiles are defined by the users, they are automat- 
ically translated into ontological instances and/or rules. These knowledge elements are used as input to the Reasoning Component and drive the service personalization process.

\subsubsection{System Integration with OSGi}

The software components of POLYSEMA and particularly of the Semantic Component are deployed on the OSGi platform in order to take advantage of the flexibility of OSGi bundles. Each of the aforementioned components is developed as an OSGi bundle, which can access other bundles' services through their registered interface in the OSGi registry.

The Multimedia Component could not be developed as an OSGi bundle, because it consists of applications running in the MHP receiver. The OSGi and MHP environments have different properties because they follow different design principles (see [21] for a relevant discussion). In order to retain full compatibility with industry standards such as MHP and DVB, and still harvest the service management flexibility of OSGi, we decided to build a delegate bundle in the OSGi platform that conceals the nature of MHP applications from the rest of the system. The MHP-based Multimedia Component communicates with the delegate bundle via the IP return channel of the receiver. The remaining OSGi-based components of POLYSEMA interact with the Multimedia Component by accessing the respective MHP-delegate OSGi service.

\section{Provision of Interactive Services}

This section lists the basic services we implemented for the POLSYSEMA prototype and gives a sample interactive application to highlight the way interactive services are executed in POLYSEMA. The services which are currently supported are:

- WWW information retrieval service: This service displays information gathered from the Internet relevant to specific elements of the media (e.g. actors, directors, scene locations etc.). The Multimedia Component receives a list of information objects retrieved from the WWW by the CRC Component. The list may contain pieces of text or images, displayed through a scrolling window interface. The user can navigate through the available information by their remote control or disable the execution of the service (Fig. 3).

- Parental control service: Parental control implements a control mechanism for blocking media access to inapropriate content. Options regarding the censoring of the inappropriate content can be one of a) Video off, b) Sound off, c) Video and sound off and d) Channel change. All options cause the suppression of inappropriate content for a time duration specified by the Reasoning Component 


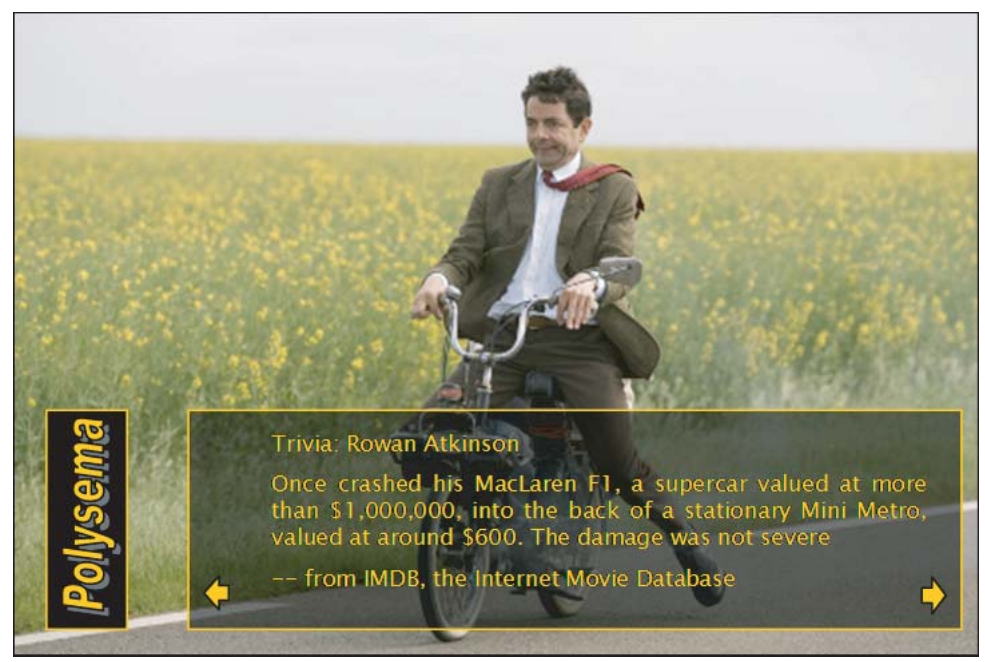

Fig. 3 Displaying information from the WWW regarding the actor.

of the system. The last option tunes the receiver to another channel in the Transport Stream defined by the user. After a time period indicated by the Reasoning Component, the receiver returns to its former state.

- PVR service: The PVR service allows the recording and playback of broadcast content. The service is activated by the receipt of a message by the Semantic Component, which instructs the automatic recording of the multimedia content for a specified time period. The user can browse a list of recorded multimedia, from which the individual recordings can be deleted, played-back, etc.

- Alerting service: This service enables the asynchronous display of messages in the form of an information alert (emergency news, sports event, etc.). These alerts can originate from different sources. An interesting case is displaying the contents of an RSS feed (Fig. 4). In this case, the CRC component is instructed to listen to specific feeds and store the respective information. Upon a change in the information included in the feed, the updated information is sent to the TV screen in form of an alert.

- Channel recommendation service: Channel recommendation aims to provide the viewer with suggestions for channels of interest to them. To accomplish this task, an external information flow is used to determine the program of the available channels. The program is described in an XML format according to the xmlTV reference specification and can be provided by the Broadcaster of the TV program. The xmlTV files contain enough information to form the basis of semantic processing at the POLYSEMA's Semantic Component. During the semantic processing, the provided information is matched against the user preferences and channel suggestions are created. The implementation of this service uses the alerting service, since the channel suggestions are displayed as special alert notices. 


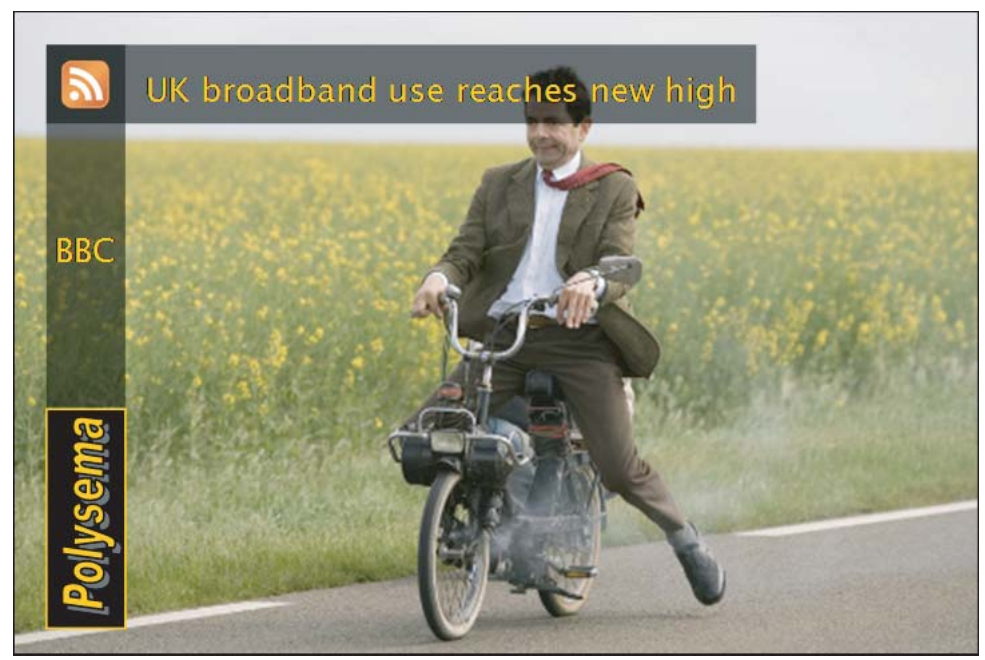

Fig. 4 Displaying an RSS Feed from BBC.

To outline how services are implemented in the POLYSEMA platform, we give an example of a service and its execution. Consider a user who includes in their profile their interest in vehicles in general. It could be requested that, in case of appearance of a vehicle in a TV program the system should collect information about it from the Web (e.g. Wikipedia). Fig. 3 depicts a screenshot of such an application.

The MPEG-7 document is assumed to contain detailed metadata that annotate the scene in which an actor drives a small motorbike (Fig. 3). A URL link to the MPEG-7 document, located at the Service Aggregator server, is transmitted through the transport stream. At the beginning of the film, the MHP application running at the receiver requests that the Semantic component downloads the MPEG-7 over the Internet. Afterwards, the Semantic component creates an MPEG-7 ontology from the document, processes the descriptions of the film's scenes to match them with the user rules and produces the respective actions. As the broadcast advances, each film scene annotated by the MPEG-7 metadata, is eventually displayed on screen. The corresponding stream event is triggered at the receiver and the MHP application requests from the Scheduler component to return the desired actions, i.e. the outcome of the preprocessing of the Reasoning component for that particular scene. Subsequently, the Scheduler supplies the desired content to the Multimedia Component through certain layout templates gathered and formatted by the Content Retrieval \& Composition Component. Then, the MHP code corresponding to the specific service is invoked in order to present the relevant information to the user. 


\section{Business Modeling Issues}

This section aims at presenting a business model which would allow the involved stakeholders to gain revenue from the introduction of semantics-aware Interactive Services by exploiting the POLYSEMA system. The existence of a sustainable business model is essential because it can leverage the practical application of our proposed system in the real world of broadcasting. To this end, we illustrate an appropriate business model in Fig. 5. In this figure, one can see the revenue ( $\mathrm{Ri}, \mathrm{i}=1 . .3$ ) and information ( $\mathrm{Ii}, \mathrm{i}=1 . .5$ ) flows between the core players and roles of this model.

The central entity in this figure is the Broadcaster. The Broadcaster is responsible for assembling any video content, application logic and metadata resources into a single digital television Transport Stream to be broadcasted to all subscribers. MPEG video files are supplied by the Media Content Provider. Application logic refers to the MHP applications required to run at the receiver in order to make semantic interactive services feasible. These applications could be provided by another market player, namely Platform Provider, but we simplify the diagram by having the Broadcaster assume the responsibility of this role. Additional responsibility of the Platform Provider is to directly provide initial installation assistance and further technical support to the subscribers.

The business model includes a class of market players called Service \& Metadata Providers. There can be a wide variety of Service \& Metadata Providers, which would allow for a broad scope of diverse applications to run on top of the basic interactive platform offered by the Platform Provider. It is not necessary that all Service Providers will provide Metadata and vice versa. Separate Players can produce only services or metadata. Nevertheless, having a single player assume both responsi-

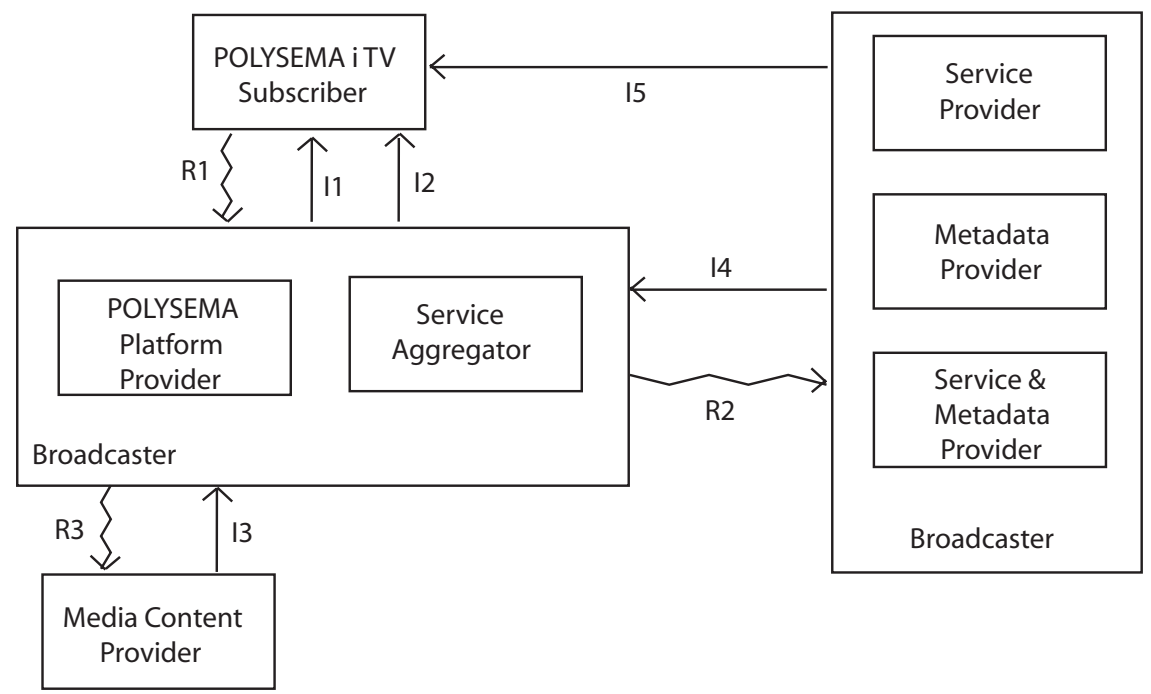

Fig. 5 Business Modeling for POLYSEMA. 
bilities will facilitate the creation of more meaningful metadata descriptions, as the descriptions will be written in correspondence to the context of specific interactive services.

The possibility of existence of several Service \& Metadata Providers indicates that a Service Aggregator player should manage the input from them. In the figure, this role is also assumed by the Broadcaster. This is because it is a good practice to let subscribers deal with a single entity, which is then responsible to distribute the revenue to the rest of the players. One of the activities of the Service Aggregator is to provide access control management. Each subscriber will have to contact the public server of the Service Aggregator to download the catalogue of services available by the platform. Without this catalogue, the receiver will not be able to provide any advanced interactive services. This catalogue can be dynamically created by the Service Aggregator, based on accounting information specific to each subscriber, thus offering a flexible, internet-based access control management scheme. A detailed description of the information flows between the various players of the business model is summarized in Table 1.

Table 1 Description of revenue and information flows

\begin{tabular}{|c|l|}
\hline Flow ID & Description \\
\hline$I 1$ & $\begin{array}{l}\text { This flow represents the Transport Stream that also contains the DVB Carousel. } \\
\text { It consists of a) the video content, b) typical broadcast information, c) the } \\
\text { MHP-related service logic and d) a link to the Service Aggregator public server. }\end{array}$ \\
\hline$I 2$ & $\begin{array}{l}\text { This flow represents the information sent to the subscriber upon login to the } \\
\text { platform. It consists a) of catalog of services that corresponds to its subscrip- } \\
\text { tion, and b) the MPEG-7 metadata file that may also depend on its subscription } \\
\text { (in case some services come with their own MPEG-7 annotations). }\end{array}$ \\
\hline$I 3$ & The video content to be delivered by the broadcaster. \\
\hline$I 4$ & \begin{tabular}{l} 
a) service data and b) MPEG-7 metadata for the broadcast program. \\
\hline$I 5$
\end{tabular} \\
$\begin{array}{l}\text { a) service OSGi part (e.g., CRC bundles), b) service-specific domain ontolo- } \\
\text { gies. }\end{array}$ \\
\hline
\end{tabular}

Finally, regarding the revenue flows we should note that various payment models and subscription types can be supported. For example, a user may subscribe for a fixed set of services or for unlimited use of services (flow R1). Alternatively, users can pay for some basic set of services and be additionally charged depending on their service usage. The flow R2 can then be implemented in a pay-per-install manner, which gives income to the Service Providers depending on the installations (for a limited period) of their services by the users. Another, Internet-oriented, revenue sharing method among the Broadcaster and Service Providers is pay-perimpression. According to this model, each provider receives commission every time a user uses its service a fixed number of times (e.g. a thousand times). 


\section{Conclusion}

The research work carried out in the context of the POLYSEMA project is driven by the great importance of metadata in providing future iTV services and the need to manage them efficiently. Moreover, the POLYSEMA platform supports applications which adapt their behavior as the content presentation advances, allowing thus for innovative iTV services. Additionally, we believe that more effective personalization can only occur if the preferences of each user are known. This can only be achieved if semantic reasoning process takes place in the end-user premises. Future research may include an even more generic framework for designing services and integrating a variety of external web resources into the TV watching experience.

In this paper we have described the design and implementation of the POLYSEMA platform as well as some sample services and some relevant business modeling issues. However, the POLYSEMA project deals with more issues peripheral to the topic of semantics-aware multimedia. Firstly, some algorithms for semantics extraction from subtitles have been designed. Such extraction involves automatic video classification from subtitles [16], ontology learning from subtitles and video summarization from subtitles. Moreover, in order to be able to test the platform with real MPEG-7 descriptions, we had to develop a new tool for MPEG-7-compliant video annotation [20]. This tool exploits ontologies for the annotation process and exports both MPEG-7 documents as well as MPEG-7 ontological instances (i.e., populates our MPEG-7 ontology). More updated information on the project results can be found in [4].

Acknowledgements This work was partially funded by the Greek General Secretariat for Research and Technology (GSRT) under the Operational Program "Information Society", co funded by the EU.

\section{References}

1. About the OSGi Service Platform - Technical Whitepaper Revision 4.0. Technical report, Open Services Gateway Initiative.

2. DVB - Implementation guidelines for Data Broadcasting, v1.2.1. ETSI TR 101202.

3. DVB - Multimedia Home Platform (MHP) Specification 1.1.1. ETSI TS 102812 V1.2.1.

4. POLYSEMA Project Web site, http://polysema.di.uoa.gr. online, 2008.

5. Implementation guidelines for DVB terrestrial services; Transmission aspects. ETSI TR 101 190, 2004.

6. The Digital Video Broadcasting Project (DVB), http://www.dvb.org/. online, 2007.

7. The Internet Movie Database, http://www.imdb.com/. online, 2007.

8. The TV-Anytime Forum, http://www.tv-anytime.org/. online, 2007.

9. Wikipedia, the free encyclopedia - http://en.wikipedia.org/wiki/. online, 2007.

10. G. Antoniou and F. van Harmelen. A Semantic Web Primer. MIT Press, Massachusetts, USA, 2004.

11. A. Lopez et al. Synchronized MPEG-7 Metadata Broadcasting over DVB networks in an MHP Application Framework. In Proceedings of Inrernational Broadcasting Convention (IBC'03), Amsterdam, Netherlands, 2003 
12. Y.B. Fernandez, J.J.P. Arias, M.L. Nores, A.G. Solla, and M.R. Cabrer. Avatar: an improved solution for personalized tv based on semantic inference. IEEE Transactions on Consumer Electronics, 52(1):223-231, Feb. 2006.

13. Stefano Ferretti and Marco Roccetti. MHP Meets the Web: Bringing Web Contents to Digital TV for Interactive Entertainment. In Proccedings of the eighth IEEE International Symposium on Multimedia (ISM'06), 2006.

14. A. Gil, J. Pazos, C. Lopez, J. Lopez, R. Rubio, M. Ramos, and R. Diaz. Surfing the Web on TV: the MHP approach. In Proceedings of IEEE International Conference on Multimedia and Expo (ICME), 2002.

15. M. Jang and J. Sohn. Bossam: an extended rule engine for the web. In Proceedings of the $3 r d$ RuleML International Workshop (RuleML 2004), November 2004.

16. Polyxeni Katsiouli, Vassileios Tsetsos, and Stathes Hadjiefthymiades. Semantic video classification based on subtitles and domain terminologies. In SAMT Workshop on Knowledge Acquisition from Multimedia Content (KAMC), Genova, Italy, December, 2007.

17. J. Martinez. Standards: Overview of MPEG-7 description tools. IEEE Multimedia, 9(3):8393, Jul-Sep 2002.

18. Serge Travert and Michel Lemonier. THE MEDIANET PROJECT. In Proceedings of Image Analysis for Multimedia Interactive Services, Portugal, April 2004.

19. C. Tsinaraki, P. Polydoros, and S. Christodoulakis. Interoperability support for Ontologybased Video Retrieval Applications. Springer Lecture Notes in Computer Science, ContentBased Image and Video Retrieval, pages 582-591, 2004.

20. George Valkanas, Vassileios Tsetsos, and Stathes Hadjiefthymiades. The POLYSEMA MPEG-7 Video Annotator.

21. A. F. Vilas, R. P. Diaz Redondo, M. R. Cabrer, J. J. Pazos Arias, A. G. Solla, J. G. Duque, M. L. Nores, and Y. B. Fernndez. MHP-OSGi convergence: a new model for open residential gateways. Wiley, Software: Practice and Experience, 36(13):1421-1442, 2006. 